\title{
Particle Filter-based Estimation of Instantaneous Frequency for the Diagnosis of Electrical Asymmetries in Induction Machines
}

\author{
Vicente Climente-Alarcon, Member, IEEE, Jose Alfonso Antonino-Daviu, Senior Member, IEEE, Ari \\ Haavisto, Antero Arkkio
}

\begin{abstract}
Fault diagnosis of induction machines operating under variable load conditions is still an unsolved matter. Under those regimes, the application of conventional diagnostic techniques is not suitable, since they are adapted to the analysis of stationary quantities. In this context, modern transient-based methodologies become very appropriate. This paper improves a technique, based on the application of Wigner-Ville Distribution as time-frequency decomposition tool, by using a Particle Filtering method as feature extraction procedure, to diagnose and quantify electrical asymmetries in induction machines, such as wound rotor induction generators used in wind farms. The combination of both tools allows tracking several variable frequency harmonics simultaneously and computing their energy with high accuracy, yielding magnitudes and values similar to those obtained by the application of the FFT in stationary operation. The experimental results show the validity of the approach for rapid speed variations, independently of any speed sensor.
\end{abstract}

Index Terms - Fault diagnosis, induction motors, time-frequency analysis, variable load, WignerVille distribution, particle filters, wind energy, $\operatorname{prognosis}^{1}$

\section{NOMENCLATURE}

$\begin{array}{ll}b w & \text { Bandwidth } \\ e & \text { Normalized energy } \\ f & \text { Supply frequency } \\ f_{b b} & \text { Rotor asymmetry fault frequency } \\ f_{k}\left(x_{k}, w_{k}\right) & \text { System transition function at time step } k \\ f_{s} & \text { Sampling rate } \\ f_{s t} & \text { Stator asymmetry fault frequency }\end{array}$

V. Climente-Alarcon, A. Haavisto and A. Arkkio are with Department of Electrical Engineering and Automation, Aalto University, P. O. Box 13000, FI-00076 Aalto, FINLAND (e-mails: viclial@ieee.org, ari.haavisto@aalto.fi, antero.arkkio@aalto.fi)

J. Antonino-Daviu is with Instituto de Ingeniería Energética, Universitat Politècnica de Valencia, Camino de Vera s/n, 46022, Valencia, SPAIN (phone: 0034-963877592; fax: 0034-963877599; e-mail: joanda@die.upv.es). 


$h_{k}$
$i_{c}$
$I F$
$p$
$q_{i}$
$s$
$W_{x}(t, \omega)$
$w_{k}$
$x_{k}$
$x^{*}{ }_{k}$
$x(t)$
$y_{k}$
$\gamma_{W, h a r}$
$\tau$
$\omega$

$\omega$
Observation function at time step $k$

Current waveform

Instantaneous frequency

Number of pole pairs

Weight of particle $i$

Slip

Wigner-Ville Distribution

System noise at time step $k$

System state at time step $k$

Estimated state of the system at time step $k$

Signal (real or complex)

Measurement at time step $k$

Wigner-Ville-based fault indicator for harmonic har

Delay

Angular frequency

\section{INTRODUCTION}

Motor Current Signature Analysis (MCSA) is the most widely used procedure [1] for the detection of induction motor's defects in the industrial environment. It consists in the identification and quantification of characteristic components in the current spectrum, whose frequency is linked to the corresponding fault and their amplitude to their severity. The effectiveness of this approach is, however, greatly curtailed when the machine operates under unbalanced supply voltages, load torque oscillations or in varying slip regime [2]. In this last case, the harmonics associated to the rotational speed appear blurred in the spectrum; thus constituting the diagnosis of machines directly connected to the grid and working permanently at variable load still a subject of research [3].

In addition, the operation of induction machines at non-constant rotating speed [4] is becoming more widespread as asynchronous generators are used in windmills due to their intrinsic advantages compared to conventional synchronous ones, among others, lower cost, easier maintenance, good dynamic behavior, selfprotection against faults and ability to work at varying speed [5].

This growing necessity of methods for diagnosing induction machines operating under variable regime has been tackled following several approaches. The extension of the Motor Current Signature Analysis (MCSA) to variable operation assuming steady state or limited and slow variations of the working conditions [6, 7] has been proposed due to the wide bibliography on the effects in the current spectrum of faults such as turnto-turn short circuits, static and dynamic eccentricity and rotor asymmetries [8]. However, the subsequent difficulties derived from the application of the Fast Fourier Transform (FFT) to short stationary or variable signals advises on rigorously implement time-frequency signal processing tools to evaluate different quantities of the machine during transient regimes [9-18]. 
Nevertheless, time-frequency processing tools have also their drawbacks, among them, higher complexity and computational costs -which often lead to un-optimized implementations- and results difficult to interpret, thus the necessity of a feature extraction procedure [19].

In this context, one of the preferred analysis tools for its relative simplicity and low computational cost has been the Discrete Wavelet Transform (DWT) [9, 11-13, 18]. However, this analysis method performs a decomposition in bands whose frequency limits are fixed by the sampling frequency (a problem tackled in $[12,13])$ and usually the defined quantification indicators integrate the energy in the whole band $[11-13,18]$, which, also computes the influence of other low-amplitude harmonics and noise presented between the limit frequencies, yielding a relatively high standard deviation of those quantification parameters and a lower sensibility to incipient faults [14].

The full capabilities of time-frequency analysis were portrayed in [15], where variable frequency harmonic tracking was initially proposed for the evaluation of components appearing in the power spectrum of wound rotor induction generators, a procedure improved in $[16,17]$. However, the wide number of sensors required by this approach makes it impractical to be implemented on a portable device, in order to be used for the diagnosis of less critical machines operating under fluctuating load/speed conditions. Only recent works [18] have highlighted the importance of the instantaneous frequency as a method for tracking a harmonic evolving in one of such bands and proposed fault detection parameters accordingly.

Furthermore, demodulation and power spectrum procedures $[3,15-18]$ have the drawback when applied in variable operation of introducing a low frequency oscillation as a consequence of the variation of the main component amplitude with load (i.e. the waveform's envelope in Fig. 3, a), in frequencies near those indicating a rotor asymmetry, hence the necessity of performing a DC extraction in [3]. Therefore, the advantage of carrying out the demodulation or the equivalent power spectrum analysis compared to the suppression of the main component by notch filters remains unclear for machines operating under variable slip.

Moreover, the use of atomic time-frequency decompositions, such as the DWT [3, 9, 11-13] or the Continuous Wavelet Transform (CWT) [16, 17] may not constitute the right choice to study the harmonic signature under fast varying speed conditions, since the windowing applied a priori limits the time-frequency resolution obtained [20]. In fact, the recurring case in the industry of differentiating between mixed eccentricity and load torque oscillations, which causes rapid speed variations and thus could be considered as an extreme case of variable speed operation, was achieved using a bilinear time-frequency decomposition tool: the Wigner-Ville distribution (WVD) [21].

The WVD has low computational costs, it can be easily implemented on DSP devices by using already optimized FFT functions, it features a high time-frequency resolution, needed to track rapidly varying harmonics and, finally, it fulfills desirable properties, such as the preservation of the energy of the signal. This feature, the accurate energy assessment of variable frequency harmonics has allowed not just the diagnosis but also proposing models for the prognosis of a rotor asymmetry in a cage machine [14]. The amount of information provided by this distribution has motivated its application in several recent works [21- 
24], especially for the study of high frequency components.

Cross terms, or artifacts that appear between the components, constitute the main drawback of the WVD. For this reason, either windowed or smoothed versions of the WVD are used, such as the Pseudo WVD (PWD) and the smoothed pseudo WVD (SPWVD), or the kernel of the distribution is modified (BornJordan, Zhao-Atlas-Marks distributions). However, these modifications come at the expense of some desirable properties $[25,26]$, especially time-frequency resolution and/or energy preservation, which are critical for incipient detection of faults by tracking rapid variable frequency harmonics.

Therefore, the approach developed in this paper consists in the use of the WVD as time-frequency decomposition tool, taking advantage of its good properties, whilst minimizing its drawbacks by suitable preand post-treatments. The first stage follows the method proposed in [27, 28], -although in this case in more demanding conditions-, that is, IIR notch and band pass filters isolate a band in which only one fault harmonic prevails, a similar process carried out using the CWT and DWT in [16] and [18]. Then, the WVD distribution is utilized to obtain the evolution of that harmonic, which yields a decomposition blurred by cross terms and, in some cases, noise. Finally, the procedure presented in $[27,28]$ is completed in this work by a novel filtering and feature extraction post-treatment, based on a Particle Filter approach, in order to distinguish the fault harmonic from the cross terms, due to the oscillatory nature of the later, yielding both an accurate depiction of the variable frequency and the energy of the fault harmonic. The resulting values are comparable to the ones obtained using the FFT under stationary conditions, even in rapid (period of one second) sinusoidal variations of speed, which allows an easy diagnosis of the fault using existing bibliography.

With the aim of validating the proposed method, this paper is structured as follows: Section III establishes the theoretical evolution under varying slip operation of the fault components that are involved in the diagnosis approach; Section IV presents the main signal processing tools utilized in this paper, its advantages and drawbacks. Section V defines the experimental procedure and the existing and proposed post- treatment methods. In Section VI, the experimental validation of the proposed approach is carried out, comparing its performance with the previous method [27, 28], and the results are discussed. Finally, section VII summarizes the conclusions of this work.

\section{EVOLUTION OF THE FAULT COMPONENTS UNDER VARIABLE LOAD CONDITIONS}

A rotor asymmetry in an induction machine induces several frequency components in the stator current, given by expressions (1) and (2) [28]:

$$
\begin{gathered}
f_{b b}=\left(\frac{k}{p} \cdot(1-s) \pm s\right) \cdot f \quad \frac{k}{p}=1,3,5 \ldots \\
f_{b b}=(1 \pm 2 \cdot s) \cdot f
\end{gathered}
$$


being $s$ the slip, $p$ the number of pole pairs and $f$ the supply frequency $(50-60 \mathrm{~Hz})$. Industrially, the most common harmonics studied in the current spectrum for the diagnosis of rotor asymmetries are the lower sideband harmonic (LSH) and upper sideband harmonic (USH) with negative and positive sign in (2) respectively. These fault harmonics evolve during normal operation around the main supply component [29].

In addition, stator electrical asymmetries cause an increase of energy in the Principal Slot Harmonics, defined by the expression (3) [30]:

$$
f_{P S H \pm n}=\left[(\lambda R) \frac{1-s}{p} \pm n\right] f
$$

where $R$ is the number of rotor bars, $n$ is an integer determining the PSH number and $\lambda=1,2, \ldots$.

Finally, in the case of wound rotor induction machines, such as the doubly-fed induction generators used in wind farms, a stator asymmetry can be monitored by tracking an also slip-dependent harmonic in the rotor current [6], given by:

$$
f_{s t}=(2-s) \cdot f \quad
$$

As presented in $[28,29,31]$, operational conditions implying changes in the slip $s$ lead to characteristic evolutions in the fault harmonics defined by the expressions (1), (2), which, studied by suitable timefrequency decomposition tools, can be used to diagnose the defect. However, in this paper the conditions are more demanding, since the fault harmonic evolves in a narrower range (slip between $3-8 \%$ compared to 0 $100 \%$ during a direct startup) and thus closer to the main current component.

\section{DATA PROCESSING TOOLS}

In this Section the main data processing tools used in the proposed diagnosis procedure are introduced.

A. The Wigner-Ville Distribution

The Wigner-Ville distribution (WVD) is a particular case of the Cohen class distributions, defined as:

$$
W_{X}(t, \omega)=\frac{1}{2 \pi} \int_{-\infty}^{\infty} x\left(t+\frac{\tau}{2}\right) \cdot x^{*}\left(t-\frac{\tau}{2}\right) e^{-j \omega \tau} d \tau
$$

being $x(t)$ the analytical signal of the real signal and $x^{*}$ the conjugate of $x$. It remains always real although its output can reach negative values at some points of the distribution [26].

The Wigner-Ville transform presents several advantages for the purpose of this paper: high resolution, fulfilling of marginal conditions (6), (7); mathematical equivalence of the first conditional moments to meaningful quantities (8) and relatively fast computation.

Its high resolution compared to other procedures [12] is a consequence of not limiting a priori the length of 
the signal by windowing. In addition and contrary to other energy distributions [21, 22] with application in diagnosis, the WVD preserves the energy of the signal, total and marginal [25, 26]:

$$
\begin{array}{r}
|X(\omega)|^{2}=\frac{1}{2 \pi} \int_{-\infty}^{\infty} W_{x}(t, \omega) d t \\
|x(t)|^{2}=\int_{-\infty}^{\infty} W_{x}(t, \omega) d \omega
\end{array}
$$

where the values $|x(t)|^{2}$ and $|X(\omega)|^{2}$ can be interpreted as energy densities in time and frequency, respectively. This enables the integration of the energy directly from the WVD output. Furthermore, the WVD also provides a method to mathematically compute the instantaneous frequency [26] by averaging all frequencies $\omega$ present in the distribution plane at a time $t_{c}$.

$$
\frac{d \varphi}{d t}=\langle\omega\rangle_{t_{c}}=\frac{1}{\left|x\left(t_{c}\right)\right|^{2}} \int_{-\infty}^{\infty} \omega W_{x}\left(t_{c}, \omega\right) d \omega
$$

However, when applied to the analysis of multicomponent or non-monotonic signals, the use of this tool is refrained by the appearance of oscillatory interferences, among those components, at time instants and frequencies in which there should be no energy. For this reason, the WVD is computed on the analytic signal obtained from the Hilbert transform [26] of the real signal. Often, a kernel function is added in the computation of the WVD to further smooth the remaining interferences, however, such modifications affect desirable properties, such as (6), (7), (8) [25], reduce the time-frequency resolution or increase the computational burden.

\section{B. $\quad$ Particle filtering}

Particle filtering, or Sequential Monte Carlo method, is a powerful tool to estimate the state's probability density function (PDF) for systems having non-linear state or measurement functions and/or their variables being not normally distributed (Gaussian) [32]. It is based on recursive Bayesian estimation, in which the PDF describing the actual state of the system is discretized as a set of weighted particles and computed in three stages [33]:

Prediction: the system transition function yields the estimated actual state of the system, according to the PDF of the last state and the system noise.

Update: that prediction is weighted by the new measurement and the observation equation via the Bayes rule.

Resampling: to avoid degeneracy, the particles are resampled according to their weight.

Mathematically, for a system having a transition function $f_{k}$ : 


$$
x_{k+1}=f_{k}\left(x_{k}, w_{k}\right)
$$

being $w_{k}$ a white noise with zero mean, independent of past and current states whose PDF is known, and an observation function $h_{k}$

$$
y_{k}=h_{k}\left(x_{k}, v_{k}\right)
$$

where $v_{k}$ is a white noise with zero mean, independent of past and current states whose PDF is known, the problem of estimating the PDF for the current state $x_{k}$ can be solved, by recursively updating the prediction $p\left(x_{k} \mid D_{k-1}\right)$ (11) of the actual state, once a new measurement $y_{k}$ becomes available, using the Bayes rule [34]:

$$
p\left(x_{k} \mid D_{k}\right)=\frac{p\left(y_{k} \mid x_{k}\right) p\left(x_{k} \mid D_{k-1}\right)}{p\left(y_{k} \mid D_{k-1}\right)}
$$

The prediction $p\left(x_{k} \mid D_{k-1}\right)$ is obtained, given the set of measurements $D_{k}=\left\{y_{i}: i=1,2, \ldots k\right\}$ and the initial state PDF $p\left(x_{1}\right)$, by:

$$
p\left(x_{k} \mid D_{k-1}\right)=\int p\left(x_{k} \mid x_{k-1}\right) p\left(x_{k-1} \mid D_{k-1}\right) d x_{k-1}
$$

being

$$
p\left(x_{k} \mid x_{k-1}\right)=\int p\left(x_{k} \mid x_{k-1}, w_{k-1}\right) p\left(w_{k-1} \mid x_{k-1}\right) d w_{k-1}
$$

where $p\left(x_{k} \mid x_{k-1}, w_{k-1}\right)$ is computed using the system transition function $f_{k}$ and $p\left(w_{k-1}, x_{k-1}\right)$ is assumed to equal $p\left(w_{k-1}\right)$;

Since the analytical solution of this recursive Bayesian estimation problem is usually not available, a set of random samples $\left\{x_{k-1}(i): i=1,2, \ldots, N\right\}$ called particles is obtained from the PDF of $p\left(x_{k} \mid D_{k-1}\right)$ and propagated through the stages of prediction, update and resampling in the following way:

Prediction: an estimation of the current state $x_{k}{ }^{*}$ is obtained by processing the set of samples $x_{k-1}$ by the system function $f_{k}$ :

$$
x_{k}^{*}=f_{k-1}\left(x_{k-1}(i), w_{k-1}(i)\right)
$$

where $w_{k-1}(i)$ is a sample extracted from the system noise PDF $p\left(w_{k-1}\right)$.

Update: when a new measurement $y_{k}$ is available, a weight $q_{i}$ for each sample reflecting its likelihood is 
obtained by:

$$
q_{i}=\frac{p\left(y_{k} \mid x_{k}^{*}(i)\right)}{\sum_{j=1}^{N} p\left(y_{k} \mid x_{k}^{*}(j)\right)}
$$

A resampling process that eliminates the samples with lower weight and multiplies the rest according to theirs, yields a distribution of particles that estimates the PDF $p\left(x_{k} \mid D_{k}\right)$ [34].

Although tracking has been one of the applications of the Particle Filtering method since their first studies [34], the approach followed in this paper is different. In this case, the WVD computed for a specific time is considered as the PDF of the state given the measurement $p\left(y_{k} \mid x_{k}^{*}(i)\right)$. The existence of negative probabilities is tackled by considering an average value of the distribution in a determined interval centered at the value of each particle, thus the oscillatory terms, the cross terms, are neglected by the algorithm, gathering the particles around the true fault harmonic. No smoothing in time or frequency is performed by this feature extraction method; thus preserving all the properties of the WVD.

\section{V. $\quad$ EXPERIMENTAL PROCEDURE}

The procedure for validating the proposed approach is presented in this section. For comparison purposes, two data processing techniques have been used, the proposed PF-based approach presented in this work and the one introduced in [28] and applied in [27] to the detection of the rotor asymmetry in a wound-rotor induction generator operating under variable speed.

A.

Experimental setup

The experimental setup consists of two motors of the same characteristics coupled back-to-back (Fig. 1). Their characteristics are: star connected, rated frequency, $60 \mathrm{~Hz}$; rated voltage $\left(\mathrm{U}_{\mathrm{n}}\right), 380-415 \mathrm{~V}$; rated power $\left(\mathrm{P}_{\mathrm{n}}\right), 22 \mathrm{~kW} ; 2$ pole pairs; stator rated current $\left(\mathrm{I}_{1 \mathrm{n}}\right), 41 \mathrm{~A}$; rated speed $\left(\mathrm{n}_{\mathrm{n}}\right), 1700 \mathrm{rpm}$ and number of rotor bars, 40. Two rotors, one healthy and another one having a broken bar, were tested in the same stator. The rotor electrical asymmetry was reproduced drilling a bar to its full depth near its junction with the short circuit ring

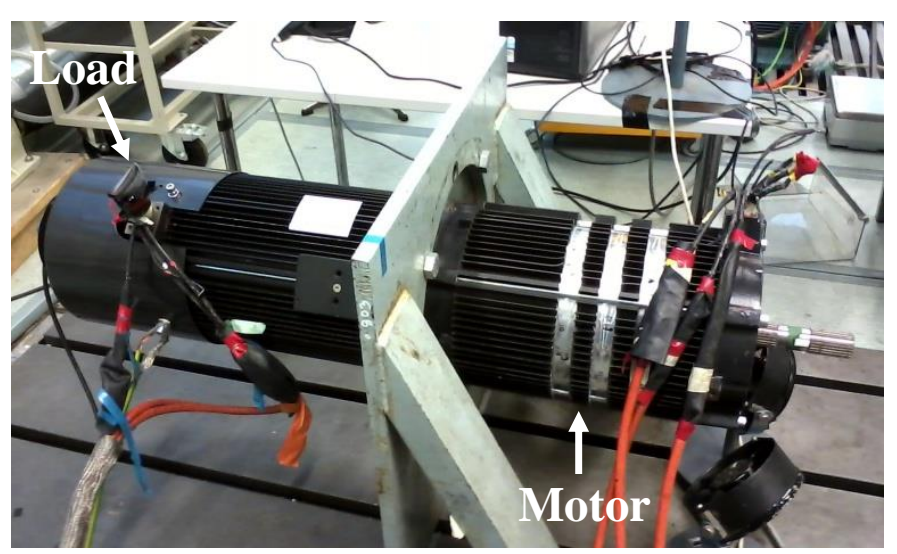


Fig. 1. Experimental test stand comprising two motors of the same characteristics coupled back-to-back, one acting as a load (left-hand side, with fan and cover) and the other as motor (right-hand side, no fan attached to it).

The tested motor is connected to the grid (frequency $50 \mathrm{~Hz}$ ) and $300 \mathrm{~V}$ through an autotransformer, whilst, in order to achieve the variable load operation, the motor acting as a load is fed from an ABB ACSM1 inverter. This inverter was commanded using an analog input connected to a waveform generator. Sinusoidal waves having a period of 4 seconds and amplitudes of 50\% (Fig. 3, a1), 75\% (a2) and 100\% (a3), and 2 and 1 second with amplitude of 100\% were programmed. The slip variation range accounted from 3 to $8 \%$. This setup permits performing fixed sequences of speed fluctuations (see Fig. 9 (b)); so being possible to test the machine under different fault conditions whilst keeping for all the tests the same variable load profile, and thus allowing a direct comparison of the results.

The current in one phase used for diagnosis (Fig 3) is captured by a LEM LT 1005 current transducer, with primary nominal RMS current of $1000 \mathrm{~A}$, overall accuracy of $\pm 0.4 \%$, linearity $<0.1 \%$, response time $<1 \mu \mathrm{s}$ and frequency bandwidth up to $150 \mathrm{kHz}$. The secondary current is converted to voltage by a shunt yielding an overall conversion ratio of $1 \mathrm{mV} / \mathrm{A}$. The resulting signal, as well as the rotational speed are recorded by a DEWETRON digital oscilloscope with a sampling frequency of $f_{s}=5,000 \mathrm{samples} / \mathrm{s}$ and a resolution of 16 bits.

The test procedure consists of commanding the inverter to turn the loading motor to near the synchronous speed at grid's frequency $(1500 \mathrm{rpm})$ and then slowly increase the voltage on the tested motor, using the autotransformer, to its equivalent at $50 \mathrm{~Hz}$, that is, $300 \mathrm{~V}$. Next, the wave generator is activated and the inverter input switched to the analog one. The frequency supplied by the inverter is reduced following the waveform captured through its analog input, slightly braking the shaft and thus loading the tested motor on a variable cycle. Since both motors are similar, the rotational speed of the shaft at each instant is equally distant from both synchronous speeds, that is, for achieving an operation point of $1410 \mathrm{rpm}$, the inverter must be commanded to turn the load motor in the same direction as the tested one at a speed of $1320 \mathrm{rpm}$, or equivalently, to an electrical frequency of $44 \mathrm{~Hz}$.

\section{Previous data processing by computation and smoothing of the IF}

The data processing procedure already presented in $[27,28]$ is summarized in the following steps (Fig. 2):

1) A pre-treatment by which IIR filters are used to isolate a band where the evolution of a fault harmonic will prevail.

2) The computation of the WVD on the analytical signal of the residue obtained from the previous step using [25].

3) A post-treatment based on the calculation of the instantaneous frequency from the WVD by (8), smoothing the high oscillating result (Fig. 4 (a)) by a low pass filter and integrating the energy in its 
vicinity (16):

$$
e_{I F, b w}=\sum_{t} \sum_{f_{b i n}=I F-b w / 2}^{I F+b w / 2} W_{x}\left(t, f_{b i n}\right)
$$

where $I F$ is the smoothed instantaneous frequency, and $b w$ the frequency range of integration. Since (16) evaluates the energy of the fault harmonic, a quantification index of the fault $\gamma_{\mathrm{W} \text {,har }}$ can be obtained comparing this result with the total energy of the original waveform:

$$
\gamma_{W, h a r}=10 \cdot \log \left[\frac{2 \sum_{t} i_{c}^{2}}{e_{I F, b w}}\right]
$$

being $e_{I F, b w}$ the energy integrated by (16) and normalized, and $i$ is the instantaneous value of the current waveform for the same time range used in (16).

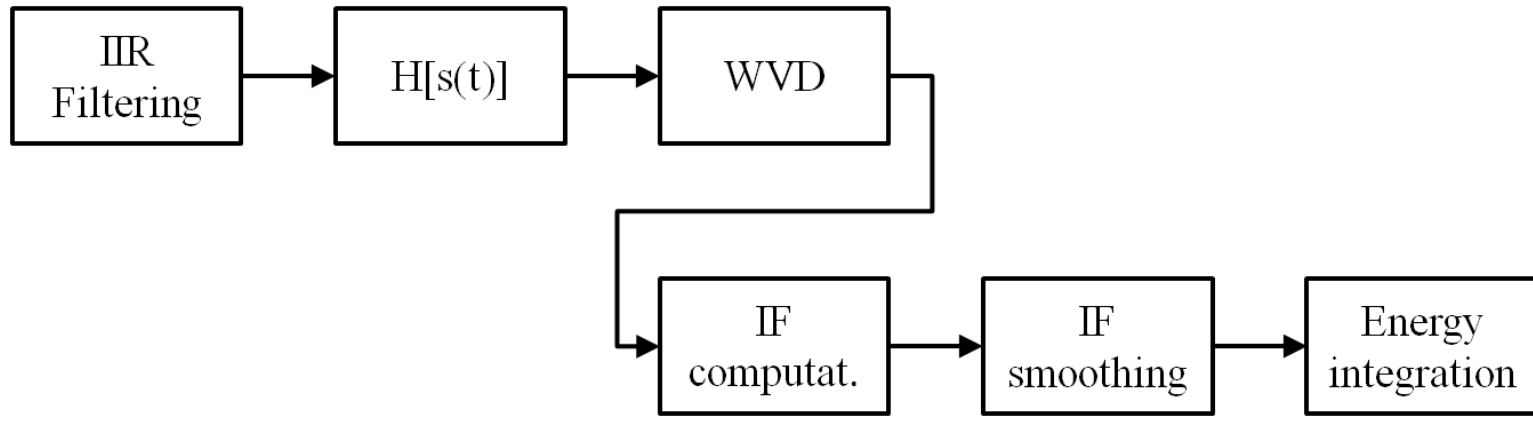

Fig. 2. Previous data processing method using IF computation and smoothing.

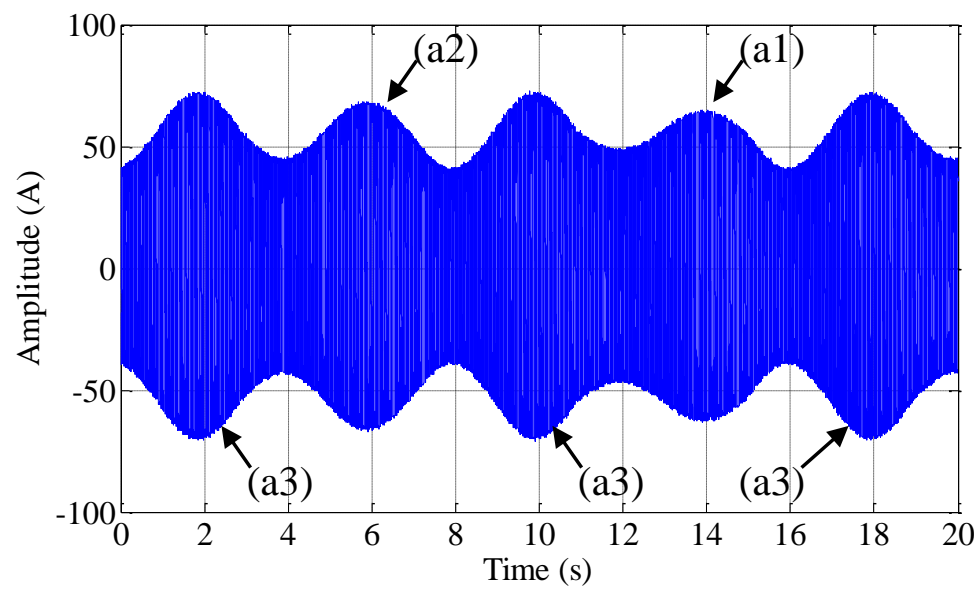

(a) 


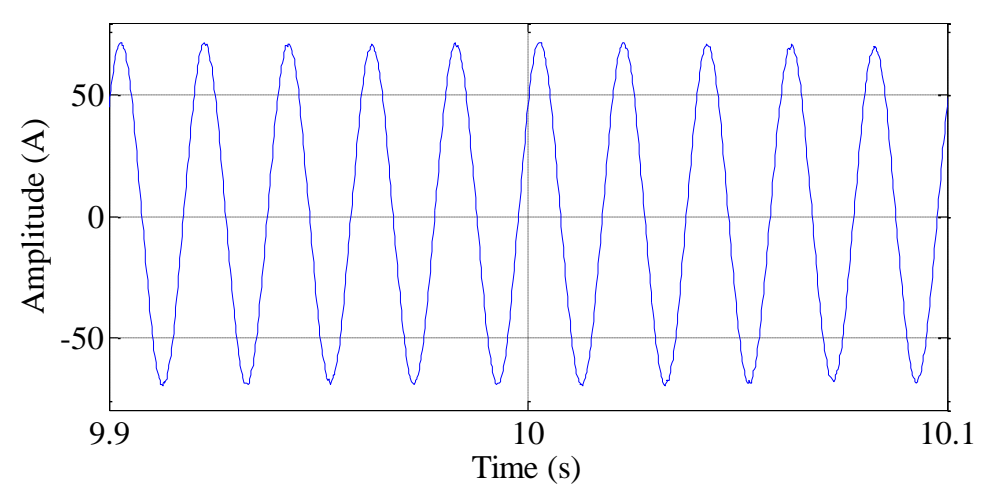

(b)

Fig. 3. Waveform captured during the variable load cycle: stator current of a motor having one broken bar, (a) entire variable load cycle, (b) detail around the $10^{\text {th }}$ second.

$C$. Proposed data processing by Particle Filtering-based estimation of the IF

The computation of the instantaneous frequency using the Wigner-Ville (5) or the Hilbert transform yields a heavily oscillating result, as it is shown in Fig 4 (a). Therefore, in $[18,27,28]$ is proposed the use of a low pass filter in order to smooth it, however, this comes at the expense of adding a delay that does not allow to track a fault harmonic during fast variations of slip, as it is shown in Fig. 7.

Thus, in order to get full advantage of the suitable properties of the WVD it is necessary to devise a procedure that allows tracking fast varying harmonics not being so sensitive to noise as the mathematical computation of the instantaneous frequency is. The procedure should ideally be able to take into account the inertia of the system, that is, given a determined harmonic's frequency, in the next time step only values close to that frequency are feasible. Moreover, the method should be flexible enough to accommodate complex working sequences, such as instants with no load, or even track multiple harmonics.

For these purposes, the harmonic tracking procedure already presented in $[27,28]$ is improved in this work (Fig. 5) by substituting the computation of the instantaneous frequency in stage 3) by a Particle Filteringbased approach performing a tracking of a target, in this case the instantaneous frequency of the fault harmonic. For the prediction stage, a one-dimensional second order model dynamic model is utilized [35]:

$$
\begin{aligned}
& x_{k}=\left(\begin{array}{ll}
\frac{d \varphi}{d t} & \frac{d^{2} \varphi}{d t^{2}}
\end{array}\right)^{T} \quad w_{k}=\left(\begin{array}{ll}
w_{I F, k} & w_{d I F, k}
\end{array}\right)^{T} \\
& x_{k}=\Phi x_{k-1}+w_{k}
\end{aligned}
$$




$$
\Phi=\left(\begin{array}{cc}
1 & d t \\
0 & 1
\end{array}\right)
$$

where the system noise $w_{k}$ is modelled as normal distributions whose standard deviations are 0.8 and 0.01 . The value of $p\left(x_{0}\right)$ is taken as the uniform distribution.

For the updating stage, each newly computed slice of the WVD at time $t_{k}$ is treated as the PDF $p\left(y_{k} \mid x_{k}{ }^{*}\right)$. The fact that the Wigner-Ville Distribution yields negative values is tackled by carrying out an averaging in the vicinity $( \pm 0.6 \mathrm{~Hz})$ of each particle value when computing their likelihood $p\left(y_{k} \mid x_{k}{ }^{*}\right)$, being rounded to zero the remaining negative results, thus, after resampling, the particles will tend to gather around the averaged higher valued areas of the distribution, ignoring the oscillatory cross terms, that in occasions can reach higher values. Their mean indicates the frequency of the fault harmonic (Fig 4 (c)). In this sense, the procedure also performs an averaging, as the computation of the instantaneous frequency by (8), but in this case all the cross terms and noise are neglected. The resulting application of this instantaneous frequency's estimation method is shown in Fig. 4 (b). Compared to Fig. 4 (a) the tracking is smoother and no delay (Fig. 6) occurs. 


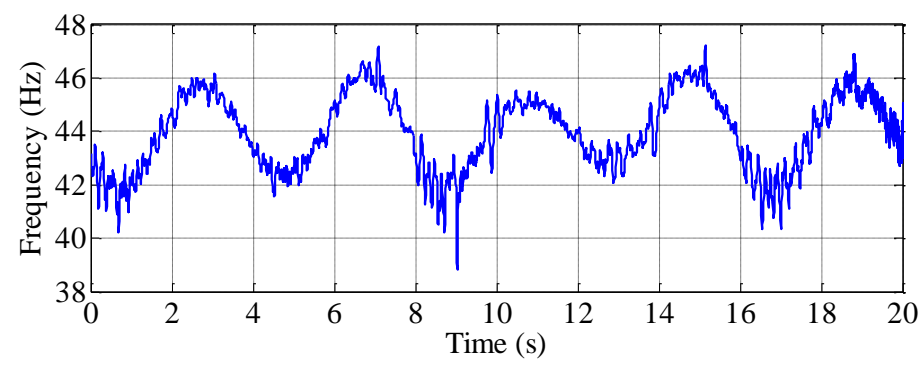

(a)

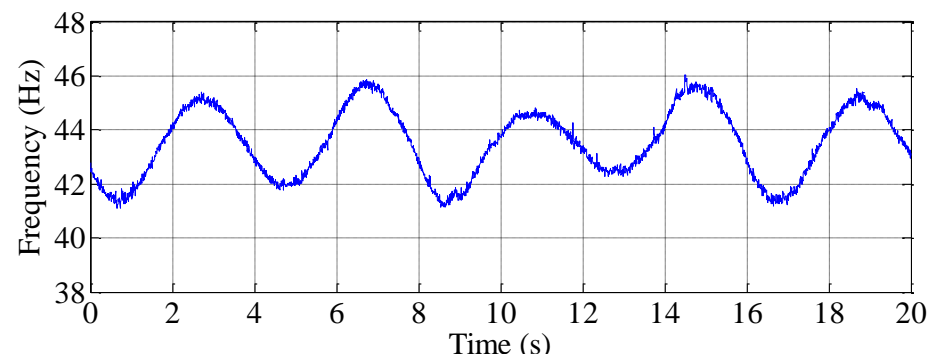

(b)

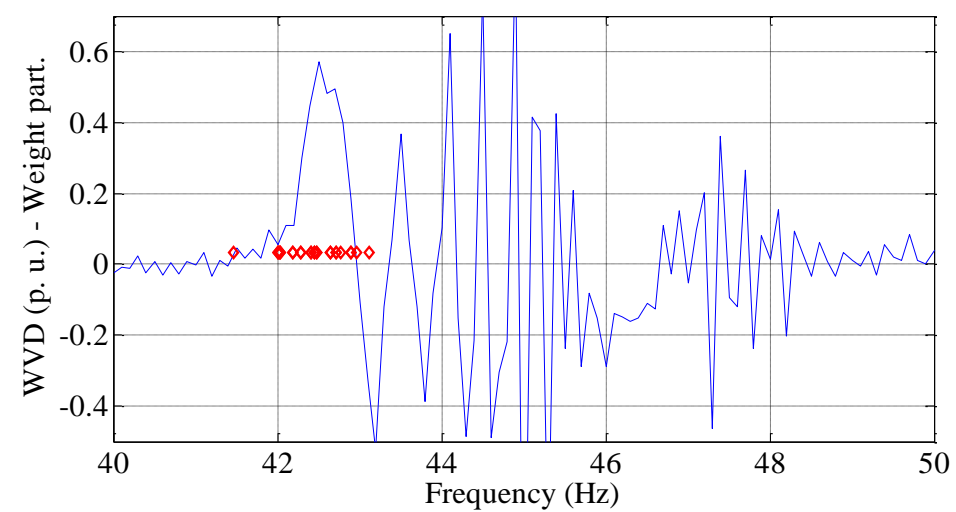

(c)

Fig. 4. (a) Instantaneous frequency computed by (8) (not low-pass filtered), (b) estimation of the instantaneous frequency using the PF approach, (c) WVD slice showing the value of the distribution in frequency (blue line) and the position and weight of the 30 particles (red diamonds) after the resampling process.

Therefore, the integration of the energy around the instantaneous frequency by (16) can be performed in a narrower bandwidth $b w$, yielding (17) a more accurate value.

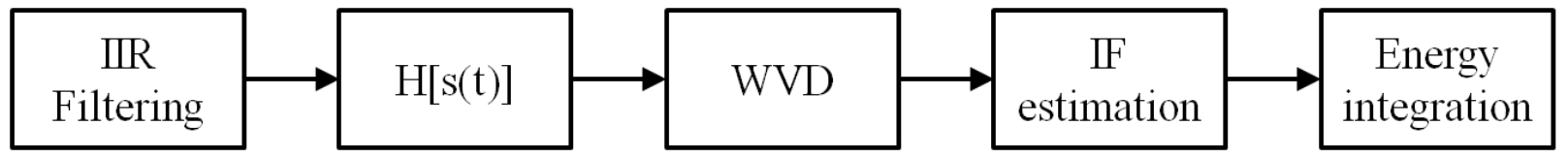

Fig. 5. Proposed method using IF estimation by PF.

VI.

RESULTS AND DISCUSSION

In order to carry out the validation of the diagnosis procedure presented in this work, the first two points of 
this section are dedicated to the comparison of the existing and proposed approach for the detection of a rotor asymmetry in an induction motor directly connected to the grid and working under variable load. In the third one the proposed method is widened to the simultaneous tracking of two variable frequency harmonics during a startup.

$A$.

Harmonic tracking by instantaneous frequency computation and smoothing

Fig. 6 shows the application of the Particle Filtering method presented in the previous section to the current signal of the induction motor in healthy state during a varying load condition characterized by a cyclic speed variation, shown in Fig. 8 (b). The LSH component is not detected in the time-frequency diagram for this case, thus the estimated instantaneous frequency varies rapidly in a pattern independent of the load, a clear sign of lacking of any fault harmonic in the time-frequency box. The scale also shows that the energy present in the time-frequency diagram is negligible. A similar result is obtained computing the IF by (8) and then smoothing the result.

The measured quantification parameter (16) $\gamma_{\mathrm{W}, \mathrm{LSH}}$ is $67.5 \mathrm{~dB}$, whilst the traditional one based on the comparison of the fault harmonic amplitude and the main current component amplitude in the spectrum during stationary operation [14] yields, for this tested healthy machine, $62.9 \mathrm{~dB}$, which is a low value even for a healthy machine (that is, the secondary circuit of this motor is highly symmetrical).

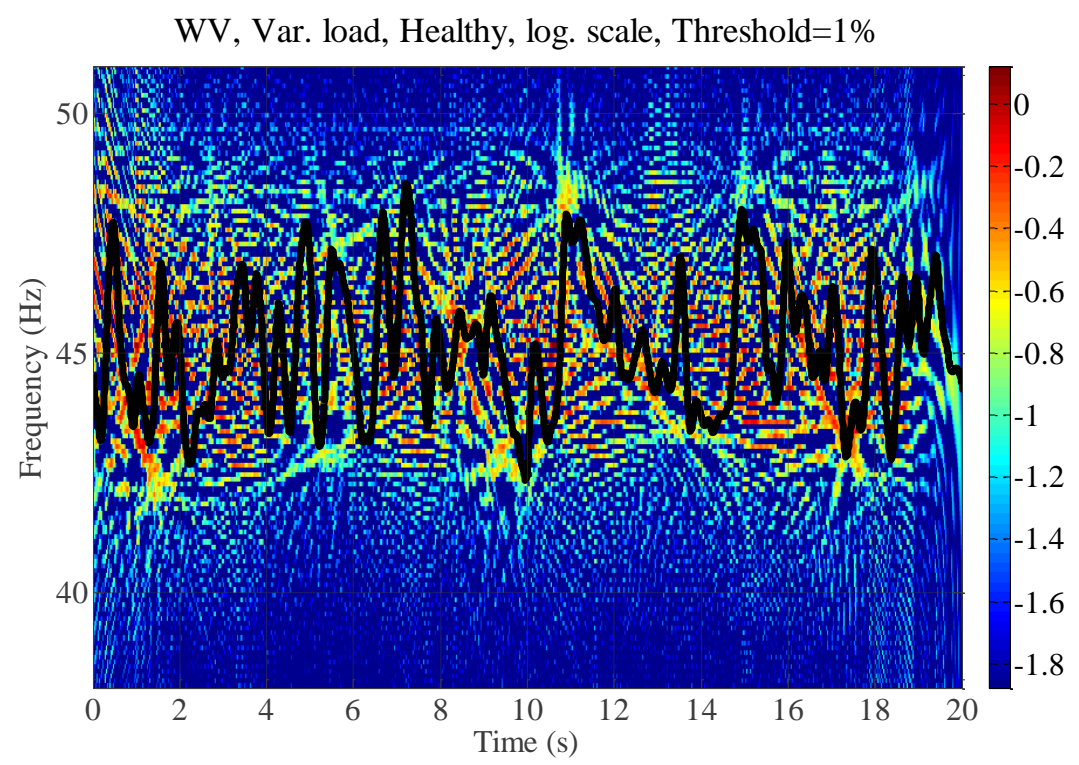

Fig. 6. WVD of the residual obtained from the filtering stage, healthy motor. The smoothed instantaneous frequency is represented by a black line.

Fig. 7 shows the current from the same motor now featuring a rotor with a bar breakage. Despite the relatively high number of rotor bars this motor features, the asymmetry is clearly discernible in the timefrequency distribution by the appearance of the LSH in the studied time-frequency box. The smoothed instantaneous frequency computed by the procedure exposed in [27, 28] locks on most of its evolution although a delay appears (Fig. 7, white arrows) at some instants. The integration of its energy is maximized 
by adding the energy present in a band of $2 \mathrm{~Hz}$ centered on its path, yielding a value for the quantification parameter $\gamma_{\mathrm{W}, \mathrm{LSH}}$ of $47.72 \mathrm{~dB}$. The quantification index calculated through the traditional approach that studies the spectrum in full load stationary operation is $42.15 \mathrm{~dB}$.

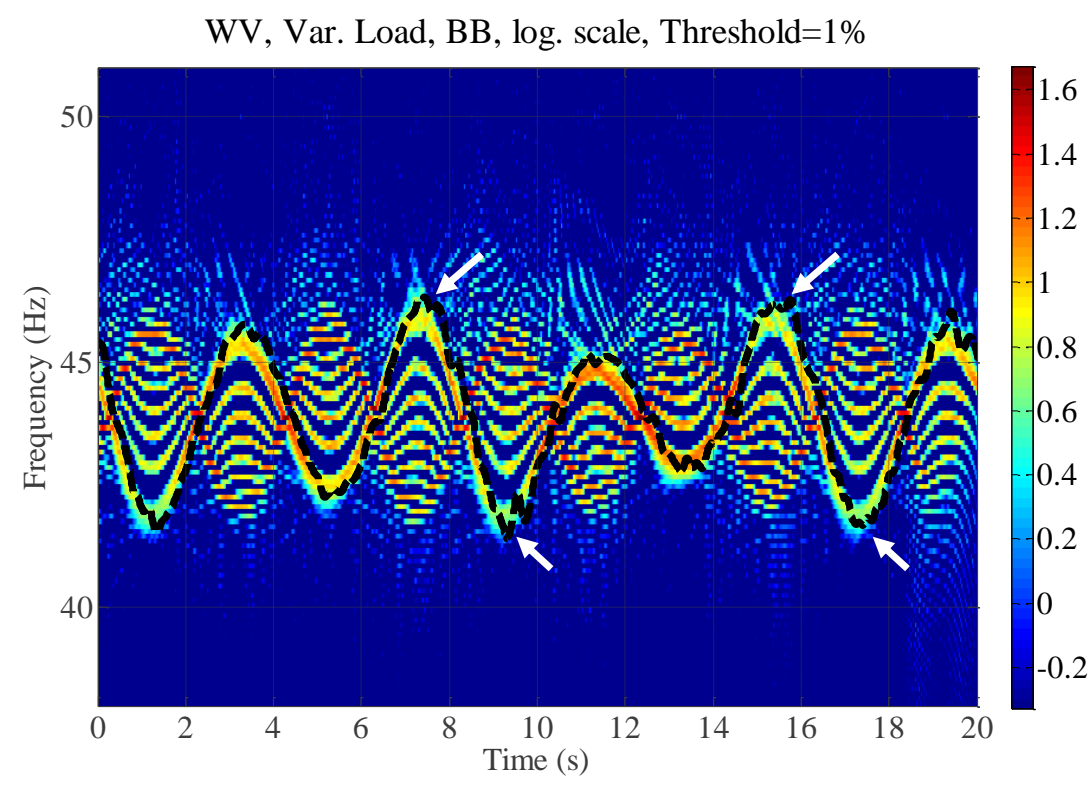

Fig. 7. WVD of the residual obtained from the filtering stage, induction motor having a broken bar. The smoothed instantaneous frequency tracking the LSH is represented by a dashed black line. The white arrows show the delay at some points of the tracking.

\section{B. Harmonic tracking by Particle Filtering-based frequency estimation}

Fig. 8 presents the result of applying the proposed PF approach to the estimation of the instantaneous frequency. As it was shown in Fig. 4, in this case no delay appears, and thus the bandwidth for energy integration can be reduced to $0.8 \mathrm{~Hz}$. The average value of the $\gamma_{\mathrm{W}, \mathrm{LSH}}$ indicator after ten estimations of the instantaneous frequency by $\mathrm{PF}$ on the same signal is $46.54 \mathrm{~dB}$ with a standard deviation of $0.01 \mathrm{~dB}$. The higher value of the indicator compared to the one of previous point reflects the better tracking of the fault harmonic and the low dispersion of the repeated ten estimations, below the measurement uncertainty value of $0.021 \mathrm{~dB}$, shows the high accuracy of the PF procedure, in spite of the random generation of particles in the resampling process, once a harmonic is present in the signal. For the case of the healthy rotor, the energy indicator value is $61.45 \mathrm{~dB}$ with a standard deviation of $0.3 \mathrm{~dB}$.

Finally, Fig. 9 (a) presents the filtered time-frequency distribution, in which only the bins where the energy has been integrated are shown. Despite the low sampling rate $(156.25 \mathrm{~Hz})$ and number of particles used $(N=30)$ the evolution of the harmonic is clearly depicted, as well as its energy with high joint time- 
frequency resolution.

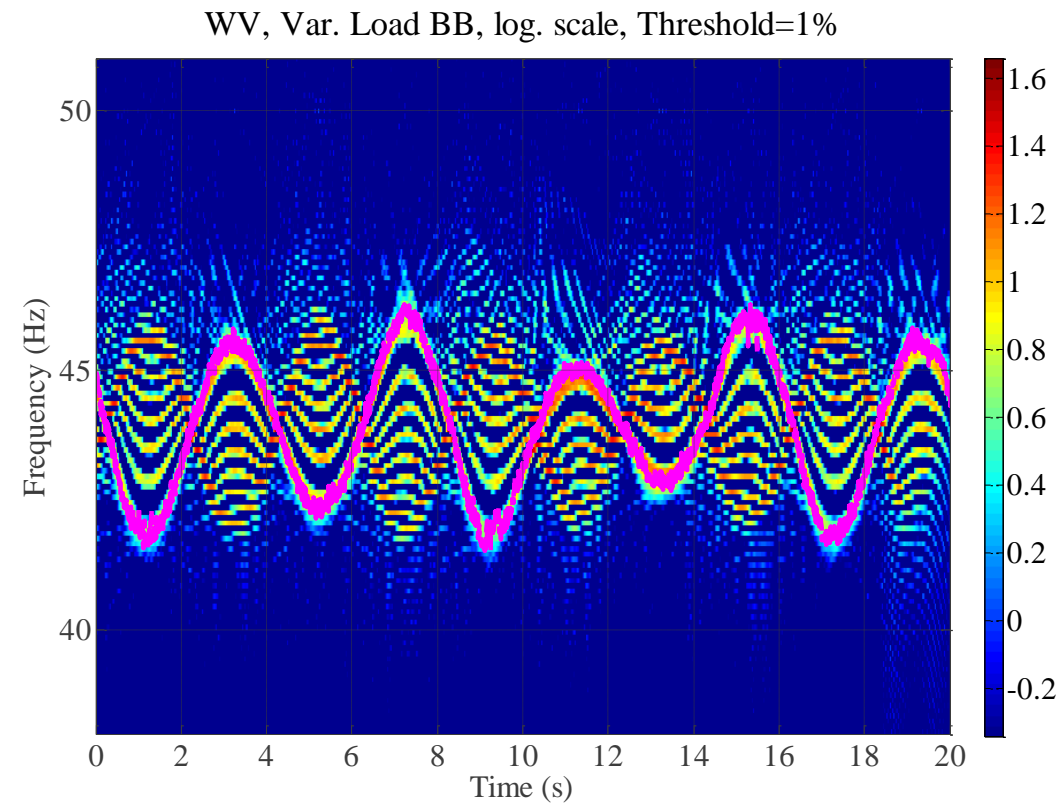

Fig. 8. WVD of the residual obtained from the filtering stage, induction motor having a broken bar. The PF estimation of the instantaneous frequency tracking the LSH is represented by a dashed rose line.

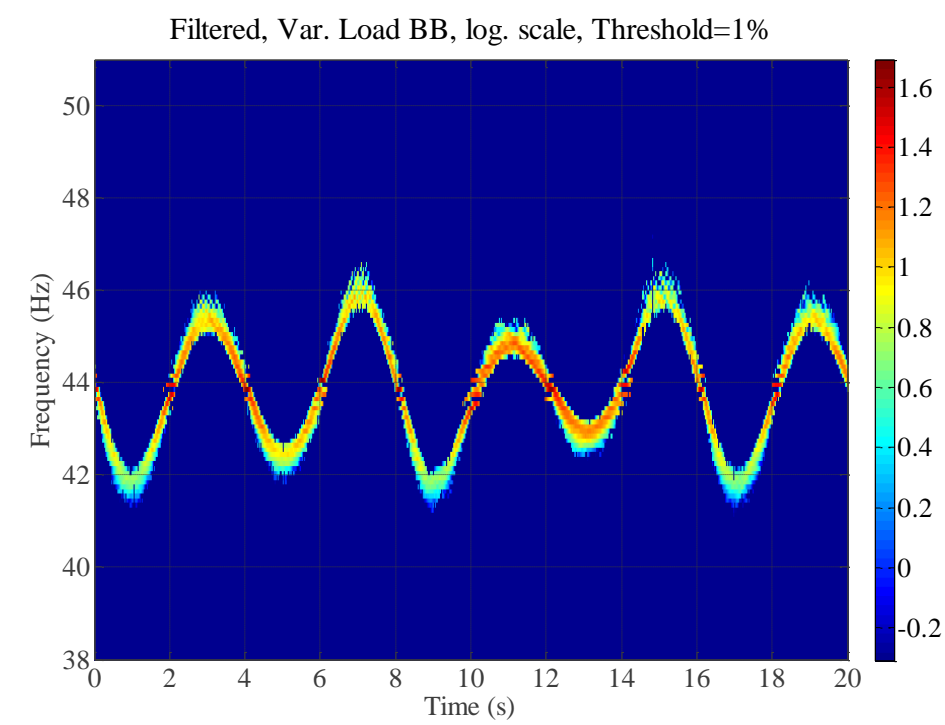

(a)

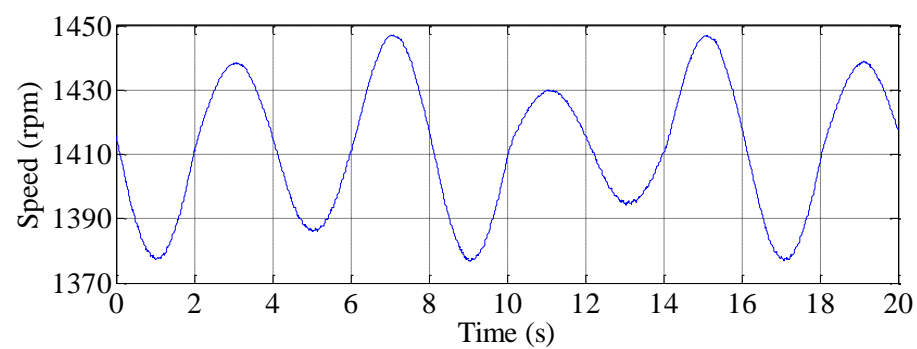

(b) 
Fig. 9. (a) Filtered time-frequency distribution of Fig. 8 showing the LSH evolution and energy density of a motor having a broken bar under variable load, (b) rotational speed during the test of Fig. 8.

Furthermore, the proposed method can also track faster evolutions of the varying frequency harmonics, such as the one shown in Fig.11 (b), in which senoids of periods 2 and 1 seconds, having the same amplitude, were programmed as loading cycle. In this case, the number of particles $N$ has been increased to 75, and the time step to $625 \mathrm{~Hz}$. Despite the high level of cross terms present in the time-frequency plot, the proposed procedure is able to track the instantaneous frequency of the component (Fig. 10 and 11 (a)) and evaluate the energy in the harmonic. The average value of $\gamma_{\mathrm{W}, \mathrm{LSH}}$ after ten PF estimations carried out on the same current waveform is $45.4 \mathrm{~dB}$ with a standard deviation of $0.01 \mathrm{~dB}$.

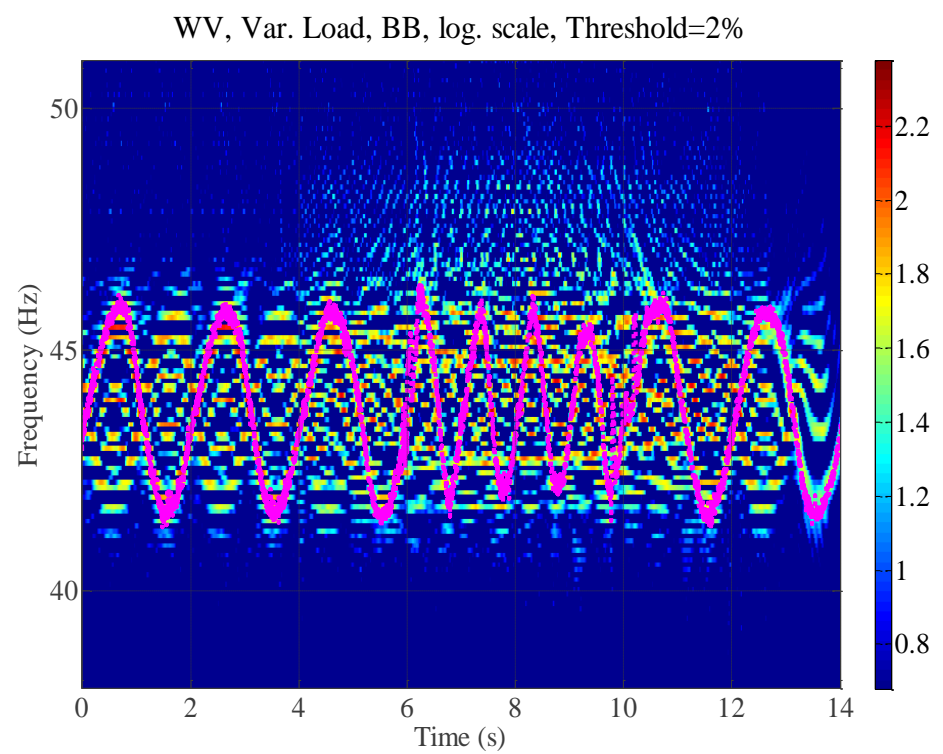

Fig. 10. WVD of the residual obtained from the filtering stage, induction motor having a broken bar. The estimation of the instantaneous frequency tracking the LSH is represented by a dashed rose line. 


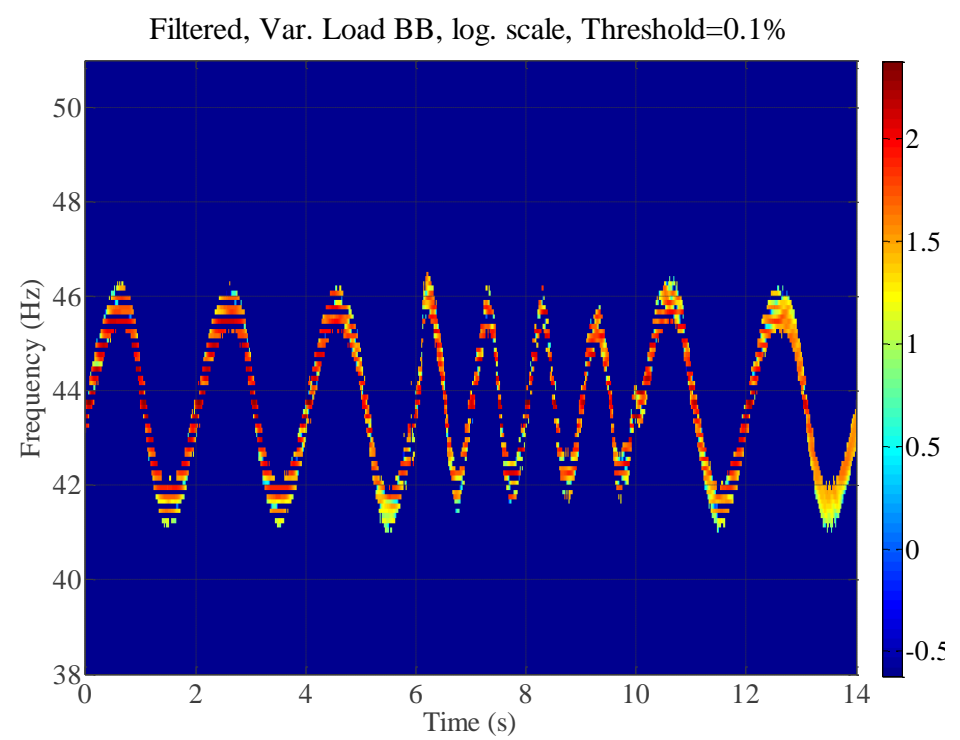

(a)

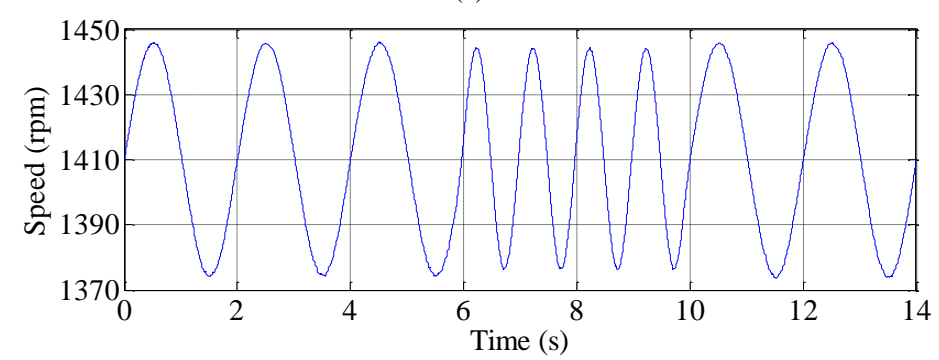

(b)

Fig. 11. (a) Filtered time-frequency distribution of Fig. 10 showing the LSH evolution and energy density of a motor having a broken bar under variable load, (b) rotational speed during the test of Fig. 10.

C.

Multiharmonic tracking by Particle Filtering-based frequencies estimation

Finally, the versatility of the proposed approach is illustrated by tracking two harmonics present in the same time frequency box, since in such cases (8) does not provide useful values. PSH tracking during a startup using the WVD was initially tried by Toliyat et Al. in [36] with the aim of sensorless speed measurement, although the energy of these components is affected by a wide variety of faults, among them, stator short circuits. However, in that early work the crossterms did not allow a successful outcome and the less accurate Short Time Fourier Transform was preferred instead.

In order to obtain the startup signal, a two pole pair, $1.5 \mathrm{~kW}$ motor, having 20 rotor bars (one broken), was coupled to a high inertia load, which permitted carrying out heavy startups of up to seven seconds. Further details of the experimental setup and pretreatment for removing constant frequency harmonics and frequencies below $50 \mathrm{~Hz}$ can be found in [28].

Fig 12 shows the result of applying the proposed approach to the last six seconds of the startup current, now having two Particle-Filtering frequency estimation blocks running in parallel. For the first one, the particles $(N=30)$ were seeded at $200 \mathrm{~Hz}$ and for the second one at $400 \mathrm{~Hz}$. The diagram shows the immediate tracking of the PSH+3 by the later group, whilst the first needs almost half a second to converge on the PSH-1 path (white arrow). The tracking and hence the energy integration (using a bandwidth of $1 \mathrm{~Hz}$ ) of 
both harmonics simultaneously is accurate during the rest of the startup, in spite of the noisy conditions increased by the high order harmonics introduced by the rotor asymmetry [28]. The values of (17) obtained are $41.48 \mathrm{~dB}$ and a standard deviation of $0.23 \mathrm{~dB}$ for the PSH +3 (upper) and $36.05 \mathrm{~dB}$ and a standard deviation of $0.21 \mathrm{~dB}$ for the PSH-1 (lower tracked component). In both cases the proposed post processing has been applied ten times to the same startup signal.

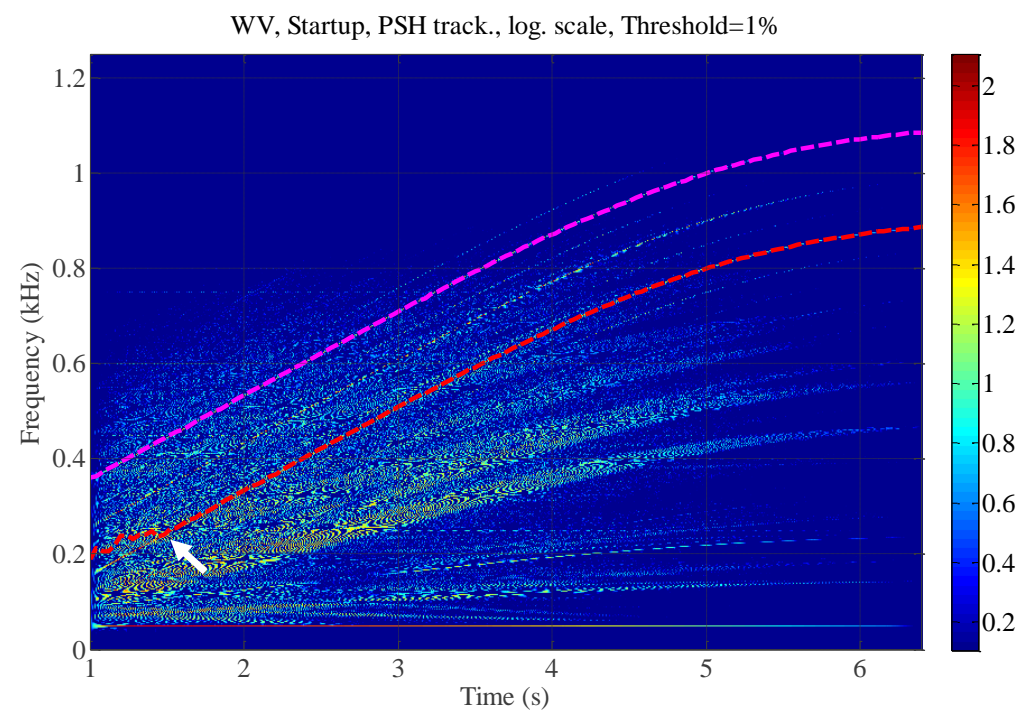

Fig. 12. PSH+3 (upper) and PSH-1 (lower) tracking during a direct-on-line startup of an induction motor having a broken bar by two different groups of particles seeded at distinct frequencies.

The results displayed in Fig. 8-12 show the ability of the combined time-frequency analysis using the WVD and the Particle-Filtering post-treatment to track fast frequency varying harmonics and accurately assess their energy. The WVD features a high joint time-frequency energy resolution, critical for this purpose, whilst the PF provides a procedure to neglect the cross terms, due to its oscillatory nature, yielding a precise estimation of the instantaneous frequency that increases the accuracy of the energy integration. This result paves the way to the study of energy phenomena in rotors during short speed transients.

As a summary, the following improvements in comparison with the previous techniques have been achieved:

1) It provides a better time-frequency resolution than using windowing techniques, and allows the tracking of fast oscillating harmonics, such as sinusoidal variations of up to $8 \mathrm{~Hz} / \mathrm{second}$ with a period of one second, in the vicinity of the main current component $(42-46 \mathrm{~Hz})$, with no need of demodulation or equivalent procedures, as in $[16,18]$.

2) The Particle Filtering post treatment allows a more accurate estimation of the instantaneous frequency of the fault component, avoiding the use of a low pass filter in order to smooth the result of its computation as in $[18,27,28]$ and can track several harmonics evolving in the same time-frequency map. 3) The proposed procedure integrates the energy of the fault harmonic in a narrower band, compared to [27, 28], of just $0.8 \mathrm{~Hz}$ centered on it, and not in the whole band, as it is done in [11, 12, 13], 
thus taking full advantage of the high resolution provided by the Wigner-Ville distribution in order to yield a more accurate indicator that truly assesses the energy of the component.

4) It works with just one sensor, that is, no rotational speed sensor is needed, as in [16], even for faster speed variations than in [27, 28].

5) The proposed post-treatment based in PF performs a feature extraction which yields meaningful indicators, equivalent and having similar values to those expected from the FFT analysis of the machine under stationary operation.

In addition, the characteristic fault-related pattern appearing in the resulting time-frequency maps can be easily interpreted, reflecting the speed variation cycle and thus constitutes a reliable tool for identifying the presence of the fault, and furthermore, the proposed approach, due to the flexibility offered by the Particle Filtering technique, can be extended to the assessment of complex varying load operation cycles such as those involving instants of operation at low slip.

\section{CONCLUSIONS}

This paper proposes an improved procedure for the diagnosis of electrical asymmetries in induction machines operating under continuous varying slip conditions. The technique is based on the analysis of just one current, by means of the application of a transient methodology having the Wigner-Ville distribution as time-frequency decomposition (TFD) tool and the Particle-Filtering approach as a feature extraction procedure for the analysis of the resulting time-frequency maps. This combined approach yields in a range of fast speed variations not covered by other previous technique, magnitudes and values similar to those expected from an FFT analysis in stationary regime. The procedure can be extended to the assessment of complex varying slip cycles or the tracking of multiple harmonics.

\section{REFERENCES}

[1] T. Thomson, M. Fenger, "Current signature analysis to detect induction motor faults", IEEE Ind. Appl. Magazine, July-August 2001, pp. 26-34.

[2] R.R. Schoen and T.G. Habetler., "Evaluation and Implementation of a System to Eliminate Arbitrary Load Effects in Current-Based Monitoring of Induction Machines”, IEEE Trans. Ind. Appl., Vol. 33, No. 7, Nov./Dec. 1997, pp. 1571-1577.

[3] Kia, S. H., Hénao, H., Capolino, G.-A, "Windings monitoring of wound rotor induction machines under fluctuating load conditions", IECON 2011 - 37th Annual Conference on IEEE Industrial Electronics Society, 2011, pp. 3459 - 3465

[4] W. Yang, P.J.Tavner, R.Court, “An online technique for condition monitoring the induction generators used in wind and marine turbines", Mechanical Systems and Signal Processing, Vol. 38, No.1, July 2013, pp: 103-112

[5] Y. Amirat, M.E.H. Benbouzid, B. Bensaker and R. Wamkeue, "Generators for Wind Energy Conversion Systems: State of the Art and Coming Attractions", J. Electrical Systems 3-1, 2007, pp. 26-38 
[6] A. Yazidi, H. Henao, G. A. Capolino, F. Betin, "Rotor inter-turn short circuit fault detection in wound rotor induction machines", proc. of the XIX International Conference on Electrical Machines (ICEM) 2010, Roma 6-8 Sept. 2010

[7] A. Stefani, A. Yazidi, C. Rossi, F. Filippetti, D. Casadei, G.A. Capolino, "Doubly fed induction machines diagnosis based on signature analysis of rotor modulating signals", IEEE Trans. Ind. Appl., Vol. 44, No. 6, Nov./Dec. 2008, pp. 1711-1721.

[8] L. M. Popa, B. -B. B. Jensen, E. Ritchie, I. Boldea, "Condition Monitoring of Wind Generators", Conference Record of the Industry Applications Conference, 2003. 38th IAS Annual Meeting, Vol: 3, 2003, pp. $1839-1846$

[9] H. Douglas, P. Pillay, A.K. Ziarani, "A new algorithm for transient motor current signature analysis using wavelets", IEEE Trans. Ind. Appl., Vol. 40, No. 5, Sept./Oct. 2004, pp. 1361-1368.

[10] Y. Amirat, V. Choqueuse, M. Benbouzid, S. Turri, "Hilbert Transform-Based Bearing Failure Detection in DFIG-Based Wind Turbines", International Review of Electrical Engineering, Vol. 6, No. 3, 2011, pp. 1249-1256.

[11] S. H. Kia, A. Mpanda-Mabwe, R. Ceschi, "Slip Independent Monitoring of Wound-Rotor Induction Machines," in proc of the International Symposium on Industrial Electronics (ISIE), 2010 , pp. $1485-1490$

[12] Y. Gritli, A. Stefani, C. Rossi, F. Filippetti, A. Chatti, "Experimental validation of doubly fed induction machine electrical faults diagnosis under time-varying conditions", Electric Power Systems Research, Vol. 81, No. 3, March 2011, pp. 751-766.

[13] Y. Gritli, C. Rossi, L. Zarri, F. Filippetti, A. Chatti, D. Casadei, "Double Frequency Sliding and Wavelet Analysis for Rotor Fault Diagnosis in Induction Motors under Time-Varying Operating Condition”, proc. of the 8th IEEE International Symposium on Diagnostics for Electric Machines, Power Electronics and Drives SDEMPED'11, Bologna, Italy 2011

[14] V. Climente-Alarcon, J. A. Antonino-Daviu, E. Strangas, M. Riera-Guasp, "Bar Breakage Mechanism and Prognosis in an Induction Motor", proc. of 9th IEEE International Symposium on Diagnostics for Electric Machines, Power Electronics and Drives, SDEMPED'13, Valencia, 2013, pp. 538-545.

[15] S.J. Watson, B. J. Xiang, W. Yang, P.J. Tavner and C. J. Crabtree, "Condition Monitoring of the Power Output of Wind Turbine Generators Using Wavelets", IEEE Trans. Energy Convers., Vol. 25, No. 3, Sept. 2010, pp. 715-721.

[16] W. Yang, P. J. Tavner, C. J. Crabtree, M. Wilkinson, "Cost-Effective Condition Monitoring for Wind Turbines", IEEE Trans. Ind. Electron., Vol. 57, No. 1, Jan. 2010, pp. 263-271.

[17] W. Yang, C. Little, R. Court, "S-Transform and its contribution to wind turbine condition monitoring, Renewable Energy," Vol. 62, February 2014, pp. 137-146.

[18] F. Vedreño-Santos, M. Riera-Guasp, H. Henao, M. Pineda-Sánchez, R. Puche-Panadero, "Diagnosis of Rotor and Stator Asymmetries in Wound Rotor Induction Machines Under Non- 
Stationary Operation through the Instantaneous Frequency," IEEE Trans. Ind. Electron., (DOI: 10.1109/TIE.2013.2288192).

J. Antonino-Daviu, S. Aviyente, E. G. Strangas, M. Riera-Guasp, "Scale Invariant Feature

Extraction Algorithm for the Automatic Diagnosis of Rotor Asymmetries in Induction Motors", IEEE Trans. Ind. Informat., Vol. 9, No. 1, Feb. 2013, pp. 100-108

J. Pons-Llinares, J. A. Antonino-Daviu, M. Riera-Guasp, M. Pineda-Sanchez, V. Climente-

Alarcon, "Induction Motor Diagnosis Based on a Transient Current Analytic Wavelet Transform via Frequency B-Splines”, IEEE Trans. Ind. Electron. Vol: 58, No. 5, May 2011, pp. 1530-1544

M. Blödt, J. Regnier, and J. Faucher, "Distinguishing load torque oscillations and eccentricity faults in induction motors using stator current wigner distributions", IEEE Trans. Ind. Appl., Vol. 45, No. 6 Nov./Dec. 2009, pp. 1991-2000

M. Blodt, D. Bonacci, J. Regnier, M. Chabert, J. Faucher, "On-Line Monitoring of Mechanical Faults in Variable-Speed Induction Motor Drives Using the Wigner Distribution", IEEE Trans. Ind. Electron., Vol. 55, No. 2, Feb. 2008, pp. 522-533

J.A Rosero, L. Romeral, J. A. Ortega, E. Rosero, "Short-Circuit Detection by Means of Empirical Mode Decomposition and Wigner-Ville Distribution for PMSM Running Under Dynamic Condition" IEEE Trans. Ind. Electron., Vol. 56, No. 11, Nov. 2009, pp. 4534-4547

S. Rajagopalan, J.A. Restrepo, J.M. Aller, T.G. Habetler, and R.G. Harley, "Nonstationary Motor Fault Detection Using Recent Quadratic Time-Frequency Representations", IEEE Trans. Ind. Appl., Vol. 44, No. 3, May/June 2008, pp. 735-744.

F. Auger, P. Flandirn, P. Gonçalvès, O. Lemoine, "Time Frequency Toolbox to Use with Matlab" CNRS (France) \& Rice University (USA) 1995-1996

L. Cohen, A.V. Oppenheim, Time-Frequency Analysis, Prentice Hall Signal Processing Series, New Jersey, 1995

V. Climente-Alarcon, M. Riera-Guasp, J. Antonino-Daviu, J. Roger-Folch, F. VedrenoSantos, "Diagnosis of rotor asymmetries in wound rotor induction generators operating under varying load conditions via the Wigner-Ville Distribution”, proc. of 2012 International Symposium on Power Electronics, Electrical Drives, Automation and Motion (SPEEDAM), Sorrento (Italy), 2012 , pp. 1378 1383 "Application of the Wigner-Ville distribution for the detection of rotor asymmetries and eccentricity through high-order harmonics", Electric Power Systems Research, Vol. 91, Oct. 2012, pp: 28-36

M. Riera-Guasp, J. A. Antonino-Daviu, M. Pineda-Sanchez, R. Puche-Panadero, and J. PerezCruz, "A General Approach for the Transient Detection of Slip-Dependent Fault Components Based on the Discrete Wavelet Transform”, IEEE Trans. Ind. Electron., Vol. 55, No. 12, Dec. 2008, pp. 41674180. 

circuits in the stator windings of operating motors, IEEE Trans. Energy Conv., Vol. 9 , No. 4, Dec 1994, pp. $652-658$

[31] M. Riera-Guasp, M. Pineda-Sanchez, J. Perez-Cruz, R. Puche-Panadero, J. Roger-Folch, J. A. Antonino-Daviu, "Diagnosis of Induction Motor Faults via Gabor Analysis of the Current in Transient Regime", IEEE Trans. Instrum. Meas., Vol. 61 , No. 6, June 2012, pp. 1583-1596

[32] B. E. Olivares, M. A. Cerda Munoz, M. E. Orchard, J. F. Silva, "Particle-Filtering-Based Prognosis Framework for Energy Storage Devices With a Statistical Characterization of State-of-Health Regeneration Phenomena”, IEEE Trans. Instrum. Meas., Vol. 62 , No. 2, Feb. 2013, pp. 364-376

A. Doucet, N. de Freitas, N. Gordon, Sequential Monte Carlo Methods in Practice, Springer, 2001

[34] N. J. Gordon, D. J. Salmond, A. F. M. Smith, "Novel approach to nonlinear/non-Gaussian Bayesian state estimation", IEE Proceedings-F, Vol. 140, No. 2, April 1993, pp. 107-113

[35] W. Suski, S. Banerjee, A. Hoover, "Using a Map of Measurement Noise to Improve UWB Indoor Position Tracking," IEEE Trans. Instrum. Meas., Vol. 62, No. 8, August 2013, pp. 2228-2236

[36] S. Ahmed y H. A. Toliyat, "A Novel Speed Sensorless Technique for Obtaining the SpeedTorque characteristics of Induction Motors for Commissioning," IEEE International Electric Machines and Drives Conference, IEMDC 2001, 2001. 